\title{
Sleep in Individuals with an Intellectual or Developmental Disability: Recent Research Reports
}

\author{
Amanda L. Richdale • Emma K. Baker
}

Published online: 6 March 2014

(C) Springer International Publishing AG 2014

\begin{abstract}
Sleep problems are a commonly reported complaint for individuals with an intellectual or developmental disability (DD), with prevalence generally exceeding that reported for individuals in the general population. Nevertheless, while there has been an increase in interest in sleep problems in children with autism spectrum disorder (ASD), sleep research in very young children and older adolescents and adults with ASD and sleep research in other DDs remains sparse. This review examines articles investigating sleep in DDs, including behavioural and melatonin interventions, published during 2012 and most of 2013, evaluating their contribution. Overall, the majority of publications concern sleep in ASD, and there remains a lack of research examining aetiology, impact and intervention for sleep difficulties in populations of individuals with other DDs. This is disappointing, given the high prevalence of sleep difficulties in DD populations and the negative impact they are known to have on individuals and their families.
\end{abstract}

Keywords Sleep $\cdot$ Developmental disability .

Intellectual disability $\cdot$ Autism spectrum disorder · Intervention

\section{Introduction}

Sleep problems are common in individuals with a developmental or intellectual disability (DD or ID) with higher prevalence rates than those in the general population [1, 2].

Amanda Richdale and Emma Baker are equal co-authors on this paper.

A. L. Richdale $(\bowtie) \cdot$ E. K. Baker

Olga Tennison Autism Research Centre, School of Psychological

Science, La Trobe University, Bundoora, VIC 3086, Australia

e-mail: a.richdale@latrobe.edu.au

E. K. Baker

e-mail: ek4baker@students.latrobe.edu.au
Reported rates of poor sleep range from $24 \%$ [3] to $86 \%$ [1], however, these are affected by heterogeneous samples [4] and varying definitions of sleep problems [5*0]. These definitions include parent-report or self-report (yes/no), researcherdefined criteria using parent-report, self-report, sleep diaries, actigraphy or polysomnography (PSG); exceeding cut-off scores on sleep questionnaires; or meeting the current Diagnostic and Statistical Manual of Mental Disorders [6] or the International Classification of Sleep Disorders (ICSD-2) [7] criteria for specific sleep disorders. Furthermore, many sleep questionnaires are not designed for populations with an ID or DD, and may not meet all desirable psychometric criteria [8]. This review examines the contribution of nonmedical papers published in 2012-2013 to our understanding of sleep problems and their psychosocial correlates in DD populations, that is, heterogeneous groups of individuals with an ID, or individuals with specific DDs.

\section{Search Strategy}

We examined sleep publications including PSG, actigraphy, sleep diaries and questionnaires, and behavioural interventions for sleep referring to ID and specific DDs. We excluded medically-orientated papers and pharmacological interventions other than melatonin. Sleep apnoea and sleepdisordered breathing (SDB) were included if they were reported within the range of sleep problems present, but medical papers addressing sleep in these conditions were not included. Both sleep apnoea and SDB are common in some DDs (e.g., Down Syndrome [DS], Prader Willi Sydrome [PWS]) [5••], and can compromise daytime behaviour, memory and learning $[9,10]$, as well as general health [11].

We searched Current Contents Social and Behavioral Sciences and Clinical Medicine databases, and PubMed over 22 months from January 2012, including "in press" articles. The titles and abstracts of articles and reviews addressing sleep in individuals with a DD or ID, regardless of age were 
examined. Forty-nine articles addressing sleep or sleep interventions were retained, eight of which were reviews; 31 articles addressed Autism Spectrum Disorder (ASD), and the remainder a range of DDs. One book chapter was identified, and one article was found in a reference list. Given the preponderance of papers describing sleep in ASD, we examined ASD separately from papers on other DDs. Intervention papers form the third section of this review.

\section{Developmental Disabilities}

Sleep research often includes ID of unknown origin, and a range of developmental conditions with a specific, or presumed genetic basis, including DS, fragile-X syndrome, ASD and a range of rarer conditions such as PWS, Williams syndrome (WS), and Smith-Magenis syndrome (SMS). These syndromes have specific behavioural phenotypes and comorbid medical conditions that may differentially affect sleep. For example, individuals with SMS have an inverted melatonin rhythm, the neurohormone that organises circadian physiology; almost all individuals with SMS have significantly disturbed sleep-wake rhythms [12]; while obstructive sleep apnoea (OSA) is a common, comorbid condition in DS associated with anatomical abnormalities [13] and problems with respiratory physiology [14•].

Sleep is a developmental phenomenon, with changes in sleep EEG, total sleep time (TST), napping, sleep onset, and sleep efficiency occurring across the lifespan [11]. Many DD studies include samples with wide age ranges, including individuals from early childhood through to adulthood, which has the potential to mask sleep difficulties associated with particular stages in development. Finally, an increased risk for sleep disorders is associated with a range of biopsychosocial factors that may impact negatively on the individual or their carers. Therefore, descriptions of sleep in people with a DD and these secondary associations, including physical and mental health, behaviour and cognition, and the impact on families also require consideration.

While sleep problems in children and adolescents are often chronic [15] and, thus, are likely to continue into adulthood, research on sleep in adults with a DD is limited. Research on the prevalence and treatment of sleep disturbance in adults with an ID (age 18+ years), and behaviours and health factors associated with poor sleep, over the period 1999-2011 was reviewed by van de Wouw and colleagues [16••]. The prevalence of settling, night waking and significant sleep problems ranged from $8.5 \%$ to $34.1 \%$. Sleep difficulties were associated with behavioural difficulties, health issues, medications, and psychopathology; behaviourally based interventions and attention to sleep environment showed some treatment benefits. However, as in paediatric populations, many studies used only subjective data, definitions of a sleep problem varied and there were a limited number of intervention studies, most of which were single case designs. The authors concluded that information about sleep in adults with an ID was still limited, as was the ability to provide appropriate clinical advice.

Following this review [16••], van de Wouw et al. [17•] obtained sleep actigraphy data for 301 older adults with ID, defining a sleep problem based on sleep latency and wake after sleep onset (WASO). At least one sleep problem occurred in $72 \%$ of the sample, with night waking $(90+$ minutes $)$ being most common $(63.1 \%)$. Over a third of the sample averaged 11 or more hours, time in bed (TIB), but not necessarily asleep. Other than being female, longer TIB was associated with factors related to more severe disability including older age, more severe ID, depression, living arrangements and being in a wheelchair. Longer sleep duration and better sleep efficiency were related to CNS medications. Thus, the use of objective measures and well-defined sleep criteria demonstrated a much higher rate of poor sleep in older adults with ID than might be expected from their review [16••]. Insufficient attention is paid to adult sleep-wake patterns in relation to bedtime, wake time, and TIB, which is excessive. TIB may relate to carer and care needs as opposed to sleep requirements.

The same group [18] examined the sleep / wake rhythm in 501 older adults with ID compared with 58 older adults in the general population, finding that adults with ID were more likely to have fragmented rhythms of lower amplitude and lower stability. Rhythm abnormalities were associated with health-related factors including dementia, sensory impairments, epilepsy and spasticity. This suggests that health factors, in conjunction with ID, present a greater risk for breakdown in the perception of cues that synchronise and maintain the sleep/wake rhythm in older adults with ID as compared with healthy elderly people in the general population. The sleep issues for elderly people with ID as highlighted here $[17 \bullet, 18]$ have implications for health care and consideration of activities that may promote better sleep. Physical activity was associated with more stable sleep / wake rhythms [18].

A recent review [19••] of sleep in children with a DD, excluding ASD, focussed on: (1) The frequency and type of sleep disturbance occurring in both specific and heterogeneous populations with a DD; and (2) the tools used to assess sleep. The prevalence of sleep problems across disorder groups differed little; on average, two thirds of children had a sleep problem except children with neurodegenerative disorders where the rate was 90-100\%. Multiple sleep disturbances were common, often severe, and chronic compared with typical populations. No single type of sleep disturbance was exclusively linked to a specific syndrome, but severity of brain impairment was associated with increased sleep disturbance. Based on ICSD-2 [7] sleep disorder criteria, all the studies reviewed reported insomnia (sleep onset and maintenance difficulties). Other sleep disturbances commonly 
reported for two or more DDs included: (1) SDB, (2) hypersomnia/excessive daytime sleepiness (EDS), (3) impaired circadian rhythmicity, (4) parasomnias, and (5) sleeprelated movement disorders; reduced TST was also reported. Over half the reviewed studies used questionnaires, none of which had been standardised for children with a DD or multiple disabilities. Furthermore, the definition of a sleep problem also varied across studies [19••]. The complexity of sleep problems in DD underscores the need to use standardised sleep measures and classificatory systems such as the ICSD2 to define sleep difficulties $[5 \bullet \bullet, 19 \bullet \cdot]$.

Several papers examined sleep in DS or compared sleep in children with DS to sleep in children with other DDs. Sleep problems in DS are estimated at 31-54\% [14•], which is below the average reported across DDs [19••]; additionally OSA, insomnia symptoms and EDS are common. Comorbid health-related conditions, psychiatric comorbidities, and medications may contribute to more severe sleep difficulties in DS [14•]. A comparison of sleep in 22 children with DS, all with full trisomy (21); 24 children with WS, which is associated with deletions on chromosome 7q, and 52 typically developing (TD) children using actigraphy, sleep diaries, and the Children's Sleep Habits Questionnaire (CSHQ) showed some disorder-specific differences in sleep [20•*. On actigraphy, children with DS had longer WASO, lower sleep efficiency, and more movement and sleep fragmentation than the other children, and they spent longer in bed than TD children. However, children with WS had longer sleep latencies than other children and TD children went to bed later. Children with DS also had a worse CSHQ total sleep score, including more parasomnias, sleep anxiety, SDB and night waking than other children, while WS children had worse sleep onset delay, poorer sleep duration and higher night waking scores than TD children. Thus, children with DS had the poorest sleep, with sleep latency being the most problematic issue for the WS children $[20 \bullet \bullet$.

Sleep in 100 individuals (25 per group) with DS (4.241.5 years); Cri du Chat syndrome (CDC) (3.8-28.5 years), which is associated with a chromosome $5 p$ deletion; Jacobsen syndrome (JS) (3.9-25.1 years), which is associated with chromosome 11q terminal deletion; and non-specific ID (4.0-31.6 years) were examined using the Modified Simonds and Parraga sleep questionnaire (MSPSQ). Most individuals lived with their families [21•]. Snoring was the most common problem in the three specific DDs. JS individuals had the most sleep problems overall, with significantly more sleep-related complaints than those with DS. Restlessness, limb movements and startles characterised JS sleep complaints. While severe sleep problems were uncommon, they were more prevalent in the JS group. The groups were not matched on gender, and the wide age range was problematic, with the average age of all participants being in adolescence; the age spread covers significant developmental changes in sleep. Problematic snoring in DS is consistent with SDB and OSA in DS $[5 \bullet, 14 \bullet]$.

PWS is associated with chromosome $15 q$ abnormalities of paternal origin with associated physical features, hypotonia and hypothalamic dysfunction; individuals are likely to have OSA, which may be central in origin, snoring and EDS [5••]. A small study examined the sleep of eight children with PWS (median 8.8 years) and 16 TD children (median 9.1 years) using actigraphy, a sleep diary, and sleep questionnaires [22]. Children with PWS had significantly more daytime sleepiness, shorter sleep latency, and longer WASO, but not increased night waking frequency compared to the TD children. Daytime sleepiness is consistent with previous research reporting EDS [5••]. Another chromosome $15 q$ disorder of maternal origin, Angelman's syndrome (AS), is also associated with significant sleep disturbances, including insomnia, parasomnias, breathing difficulties and daytime sleepiness [5••]. A sleep study using PSG $(n=14)$, actigraphy $(n=10)$ and the CSHQ $(n=15)$ with 16 children with AS (2-16 years) found extended sleep latency (mean $>30$ minutes), periods of night waking of an hour or more, increased sleep fragmentation, and reduced TST. Scores on the CSHQ exceeded normative values for the total score and all subscale scores [23•]. Thus while both these chromosome 15 disorders are associated with poor sleep quality, the aetiology of the poor sleep may differ, and OSA appears to be a feature of PWS but not AS.

\section{Cognition and Behaviour}

Intellectual impairment present in those with a DD can vary from profound intellectual disability to above average intellectual functioning, depending on the disability. Both significant daytime behavioural problems and more severe levels of cognitive impairment are associated with increased risk of sleep difficulties in those with a DD [3]. Research in TD populations has implicated sleep as important for cognition, executive function, memory, and academic performance [10, 24]. In particular, both SDB and OSA are detrimental to attention [25], neurocognitive functioning and academic performance in children [10, 24] and adults [26]. Thus, IQ is a potential confounding factor for sleep problems in DD populations, and there is a need to understand any impact of poor sleep on daytime functioning in individuals with a DD.

Ferndez and Edgin [9] hypothesised that the presence of disrupted sleep, resulting from SDB and OSA from a very young age in individuals with DS may compromise brain development and function, contributing to cognitive decline over time. Thus, "sleep disruption" may contribute to "pathological aging" (p. 3) in DS, in particular the neurological effects of OSA may lead to increased brain beta-amyloid deposits associated with Alzheimer's disease [9]. The impact of poor sleep (Simonds and Parragara questionnaire) and OSA on cognitive function in 29 adolescents and young adults with 
DS was examined; verbal fluency was associated with insomnia, daytime behaviour and sleepiness were associated with inhibition, and OSA was associated with both verbal fluency and inhibition [13]. More severe OSA was also related to increased BMI. Thus, poor sleep quality and OSA impact negatively on cognitive performance in DS. Treatment of sleep problems in DS may lead to improved cognitive function, including preventing later cognitive decline $[9,13]$.

In a group of 14 (all measures) to 18 (sleep questionnaire) children with WS (15.4- to 48-months), shorter night sleep was related to language development with additional variance being explained after age was accounted for [27]. The WS children slept less, woke more often, and were more likely to co-sleep than TD children; WS parents were significantly more likely to say their child had a sleep problem [27]. A decrement in learning performance (finger-tapping task) was reported for 14 children with WS (6-11 years) compared with age-matched TD children who improved [28] following sleep. This performance difference was attributed to the sleep problems evident in the WS group (actigraphy and CSHQ). However, task performance was initially much lower in the WS than the TD group and may indicate failure of the WS children to sufficiently consolidate the task during the initial learning period.

Two reviews also examined the potential effects of sleep difficulties on early development [29] or on cognitive function and academic performance [30], arguing that screening for sleep problems should occur within early intervention (EI) [29] and special education [30]. Bonuck and Grant [29] contended that significant sleep problems fell within eligibility criteria for EI as early treatment of childhood sleep problems could prevent developmental problems. While the assessment of poor sleep, the provision of parent education about children's sleep and appropriate referrals for sleep intervention within EI programs are important aims, the inclusion of disordered sleep as an eligibility criterion for EI services seems problematic as sleep disturbance is common in children under 6 years, regardless of developmental delay [31]. Despite the known associations between poor sleep and cognitive performance and academic achievement, research on the impact of improved sleep on the daytime performance of children with a DD is lacking [30]. Health risk factors (e.g., asthma, obesity) and lower socioeconomic status are also risk factors for poor sleep [30], and should be considered; these also independently contribute negatively to daytime performance [11].

\section{Health-Related Conditions}

Insomnia is also a symptom of psychopathology, particularly anxiety and depression [6, 32]. Disrupted sleep associated with shift work and OSA increases risk for medical conditions including diabetes, cardiovascular conditions, obesity, alcoholism and psychiatric disorders [11]. Disturbed sleep is also associated with chronic medical conditions including those causing nocturnal pain, epilepsy, and asthma [32]. Doran et al. [33] reviewed a range of medical and psychological factors that are associated with, or exacerbated by poor sleep, which should be considered when examining sleep in individuals with a DD. Psychological factors include mood, attention, executive function, motor skills and learning. Therefore, sleep disturbances can be harmful to health and daytime functioning, and may harm development [32]; untreated sleep issues have the potential for a range of negative medical and psychological impacts on individuals with a DD.

One health-related study [34••] examined BMI, physical activity, sedentary behaviours (screen use), sleep timing and hours slept in 410 adolescents (mean age 15.1 years) from schools or classes for those with an ID. Based on adolescent $(n=381)$ sleep and rise times, four groups were identified: $15 \%$ early bed/early rise; $43 \%$ early bed/late rise; $15 \%$ late bed/early rise; $27 \%$ late bed/late rise. Early bed/late rise adolescents averaged $10.5 \mathrm{hrs}$ sleep and late bed/early rise adolescents averaged 7.7 hours sleep with the other groups averaging about nine hours. Late sleepers had increased screen time, were more likely to be overweight or obese, and were less physically active. After adjusting for sleep length, the lowest risk for overweight plus obesity was in the early bed/early rise group, while the late bed/late rise group had the highest risk of obesity plus overweight, with rates of $13.6 \%$ and $22.5 \%$, respectively, in each group, but both groups had similar TST. Results indicated that in adolescents with ID, sleep timing is more indicative of overweight/obesity than is sleep length; early bed/early rise adolescents were more active. This is consistent with reports that the timing of sleep [35] rather than short sleep [36] may be the more important risk factor for overweight/obesity in typical adolescents.

\section{Families}

The impact of poor sleep on individuals and their families can also be significant. In particular, mothers are more likely to report problematic sleep and daytime fatigue. Mothers of TD children with sleep problems are more stressed and are at greater risk for psychopathology [37]. In children with a DD, mothers have more sleep difficulties associated with higher levels of anxiety, depression and stress [38, 39]. Parents of children with DD and sleep difficulties also report concern, not only about their child's sleep but also the disruption caused to the sleep of other family members [40].

In children with AS (2-16 years), actigraphic measurement of parents' sleep showed that night waking and sleep fragmentation were associated with child sleep duration (CSHQ), while parent stress was related to children's variable night sleep times. The child's sleep latency was associated with 
parents' daytime sleepiness and insomnia [23•]. Further, in children with WS, children's night waking was associated with poorer maternal sleep and higher depression scores [27]. Overall, poor child sleep is detrimental not only to the child with DD but also to family members, especially mothers.

\section{Conclusions}

Recent papers reinforce that poor sleep quality is a common feature of DDs, regardless of age or disorder, with the potential to have significant negative impacts on individuals and their families. Like children with a DD, older people with ID appear to be at greater risk of disturbed sleep than in the general population. While symptoms of insomnia are common across DDs, biological factors associated with some DDs predispose individuals to sleep disorders such as EDS, SDB and OSA. Furthermore, there may be disorder-specific trends in sleep difficulties, with a greater prevalence of sleep onset difficulties in WS, overall poor sleep quality with long sleep latency and lengthy WASO duration occurring in AS, and restless sleep/movement occurring in JS. While both PWS and AS are chromosome 15 disorders and are associated with daytime sleepiness, the aetiology of daytime sleepiness differs in the two disorders. Thus, while there are no clear, disorderspecific sleep problems, examining poor sleep in heterogeneous samples of individuals with an ID or DD may mask aetiological factors that have important implications for prevention and treatment.

\section{Autism Spectrum Disorder}

\section{Characterisation}

Sleep problems in ASD are common and begin at a young age [5••], but when sleep begins to diverge from that found in TD children is unknown. According to a recent longitudinal study $[41 \bullet \cdot]$ significant differences between parent-reports of sleep in children with ASD and TD children begin to emerge at the age of 30 months; however regulatory problems, including sleep, appear to concern parents even prior to diagnosis [42]. Humphreys and colleagues [41••] study showed a trend for children with ASD to have reduced TST and increased night waking episodes, which persisted into adolescence. Selfreports of sleep patterns and associated behaviours in adolescents with high-functioning autism spectrum disorder (HFASD) showed that these adolescents had more symptoms of insomnia, significantly lower sleep efficiency, more fatigue and a trend towards reduced TST compared with age-matched and gender-matched TD controls [43•]. These findings are consistent with a large parent-report study showing more problems with sleep onset, decreased TST, and daytime sleepiness in adolescents with ASD [44]. Therefore, recent findings, including self-report, continue to support that sleep problems in ASD start at a young age and persist into adolescence.

Children with ASD experience a high prevalence of sleep onset problems compared to children with other DDs, but not WASO, potentially differentiating them from other DD groups [40]. Consistent with previous research, children with ASD had significantly shorter WASO duration compared to children with ID [45], and were more likely to experience sleep onset insomnia than children with ADHD, epilepsy, and typical development [46], and slept less within a 24-hour period than children with ID and TD children [45]. Children with ASD did not differ from children with ADHD on parentreports of current and lifetime sleep problems [46], which may relate to both the high prevalence of sleep problems in ADHD [47] and the comorbidity of ASD and ADHD [48].

\section{Child Functioning and Family Impacts}

Sleep problems in children with ASD have been associated with children's challenging daytime behaviours, academic achievement, and mental health. They also have a negative impact on the sleep patterns and mental health of parents [5••]. Several new papers addressed these issues in 2012.

Both poor sleep quality and reduced TST were associated with poorer cognitive abilities, socialization, adaptive behaviours, and communication skills from infancy to adolescence in children with a diagnosis of autism or Pervasive Developmental Disorder-Not Otherwise Specified; additionally dysfunction in adaptive behaviours worsened as the severity of the sleep problem increased [49]. Nevertheless, poorer socialization and communication skills are related to sleep problems (regardless of severity) in children with ASD [50]. Extending these findings, Tudor and colleagues [51] found that sleep onset delay was the strongest predictor of an Autism Index (AI) score; longer sleep onset duration was associated with a higher AI score. Moreover, higher scores on the communication and repetitive stereotyped behaviour (RSB) domains on the ADI-R have been related to bedtime resistance and insomnia in children and younger adolescents with ASD [52]. Therefore, more severe behaviours associated with an ASD diagnosis may be associated with the manifestation of sleep disturbances or vice versa.

Mothers of children with ASD also experience poor sleep [53]. Mothers of 90 children with ASD (4-12 years) and 90 TD children of the same age participated in a study examining mothers', and children's sleep as well as mothers' mental health [54]. Children's sleep directly affected mothers' stress, sleep quality and mental health, with mothers' stress and sleep also mediating the effect of child sleep on mental health for both groups. ASD acted as a moderator of the child's sleep and maternal stress relationship and the relationship between mothers' stress and sleep, suggesting that the child's sleep is 
only one of many sources of stress for ASD mothers. Sleep in children with ASD is associated with significant behaviour difficulties [5••], while behaviour difficulties are associated with parent stress [55]. Thus, it may be that some of the variance in maternal mental health and maternal stress are accounted for by child behaviour difficulties as well as child sleep where the child has ASD.

\section{Psychopathology}

Both anxiety and depressive disorders are common comorbidities in individuals with ASD [56] and researchers report associations between sleep disturbances and internalising and externalising problems $[45,50,52]$. In children with ASD, self-reported poor sleep was significantly associated with affective problems, somatic panic, anxiety, separation anxiety, and depression scores [57•], while assessment of the predictors of CSHQ-23 scores in 1,583 children and adolescents with ASD showed that anxiety was the strongest predictor of sleep disturbance [58 ]. This relationship requires further consideration, as it is likely to be bi-directional, with poor sleep exacerbating symptoms of anxiety [58•], however it is consistent with a recent report that CSHQ predicted internalising scores in children diagnosed with an anxiety disorder [25].

\section{Cognition}

Studies of sleep disturbance in adults with ASD or the impact of sleep on cognition in ASD are uncommon, but one paper [59••] assessed the relationship between poor sleep, as measured by PSG, and daytime cognitive performance in adults with autism. Poor sleep and performance was similar between groups on a selective attention task, however, the ASD group differed on sensory-motor and cognitive procedural memory tasks. ASD participants performed worse on these latter tasks suggesting that the relationship between sleep and performance for non-verbal tasks is not necessarily linear [59••]. The authors propose that research needs to further clarify whether poor performance is related to autism diagnosis, poor sleep or a combination of both.

\section{Aetiology}

Sleep problems may be behaviourally based, be exacerbated by poor sleep hygiene practices, be related to comorbid psychopathology, or may be related to biological factors or core features of ASD [5••]. Current research investigating the aetiology of sleep disturbance in ASD is limited, but recent research is promising.

Sleep hygiene is an important consideration when examining the causes of poor sleep in DDs [60]. A new study has assessed the effect of in-room gaming systems, computers and television in boys with a diagnosis of ASD, ADHD or who were TD children [61•]. Consistent with previous research in TD children [62], the researchers found that in-room gaming systems have a negative impact on sleep regardless of childhood disability. However, in-room televisions and computers had a more severe impact on the boys with ASD than on ADHD or TD boys. The results emphasise the contribution of electronic devices to poor sleep hygiene and the need to remove such devices from bedrooms.

Several studies examined potential medical or biological factors that might contribute to poor sleep in ASD. An investigation of the relationship between serum ferritin levels and sleep disturbance in children with an ASD indicated that children with ASD had significantly lower serum ferritin levels compared to control children [63]. Additionally, levels were lower in ASD children with Periodic Limb Movement Disorder (PLMD) and sleep fragmentation. Children with ASD may not obtain optimal levels of iron from dietary intake, which may have a negative impact on their sleep, due to an association between iron intake and leg movements and restless sleep [64]. In ADHD children, low serum iron has been associated with disturbed sleep including sleep movements [65].

GI problems are commonly reported in children with ASD and children with DD [66]. Two recent studies [58•,67] reported that GI problems significantly predict sleep disturbance in children with ASD. Specifically, abdominal pain predicted sleep anxiety, while under-eating and GI symptoms predicted parasomnias in children and adolescents with ASD [67]. Further, GI problems including constipation and diarrhoea predicted CSHQ-23 total scores and sleep anxiety [58•]. Further research is required to clarify the relationship between GI problems and sleep disturbance in ASD.

Alterations in cortisol secretion have been reported in individuals with ASD [68, 69], suggesting that the stress response may be altered in $\mathrm{ASD}$; stress response is related to anxiety, which can be incompatible with good sleep quality $[11,70]$. The assessment of sensory processing, physiological stress, and behaviours in children with ASD and TD children indicated that poor sleepers (regardless of group) had increased afternoon cortisol levels and higher cortisol reactivity at each time point after a sensory challenge. The greatest difference occurred 25 to 30 minutes post-challenge [71•] and increased sensory sensitivity was associated with higher afternoon cortisol levels. The authors concluded that heightened levels of afternoon salivary cortisol may be a neuroendocrine response to sensory stresses occurring throughout the day, and subsequently related to poor sleep. Thus, heightened anxiety and sensory sensitivities may exacerbate sleep problems in children with ASD. Furthermore, sensory sensitivities have been shown to predict sleep disturbance in children with ASD [58•].

Given that ASD has a strong genetic component, with a $7 \%$ recurrent sibling risk-rate [72], whether or not poor sleep 
is familial may assist in assessing developmental risk. In a study comparing high-risk and low-risk siblings [73•], more high-risk siblings were reported by parents to have borderline clinical or clinical scores for sleep problems, anxiety, depression, somatic complaints, and aggressive behaviours compared to low-risk siblings. Further, both children with ASD and unaffected siblings were more likely to have parentreported early insomnia compared to TD children [74].

\section{Conclusions}

Given research over the past 20 years, our knowledge of sleep and ASD has not progressed as much as might be expected. Research now strongly supports the notion of increased sleep problems in individuals with ASD [5••, 75]. However, recent literature continues to focus on children and adolescents with very little being known about infant and adult sleep problems in this population. Further, investigation of the underlying aetiology of sleep problems is lacking; however, recent research is promising. In line with past research [76] and given the heterogeneity in the presentation of ASD, current research supports the notion of multiple aetiologies [76]. Sub-groups of individuals with ASD may exist based on the aetiology of the sleep disturbance. Firstly, individuals with ASD may experience sleep problems due to biological abnormalities. This is supported by research showing disrupted sleep in first-degree relatives with ASD and melatonin dysregulation in this population. Secondly, core features of ASD such as RSBs, sensory sensitivities, and increased symptom severity may disrupt sleep. Lastly, the presence of anxiety and depression may result in poor sleep. In determining the underlying aetiology, appropriate sleep intervention and treatment techniques can be developed. Understanding the evolution of sleep problems and any existing subgroups may also serve as red flags for the surveillance of young children's development.

\section{Intervention}

Sleep treatment research is lacking for individuals with DDs $[5 \bullet \bullet, 16 \bullet]$, and often lacks long-term evaluation. Stores and Stores [14•] concluded that sleep interventions for children with DS are needed in order to alleviate both family and child difficulties. Related to the issue of treatment is that of assessment, without appropriate assessment, appropriate interventions cannot be implemented. A medical assessment for any underlying health issues affecting sleep, and to assess problems such as SDB and OSA is needed and an assessment of the presenting sleep problem using valid tools is required; a screening questionnaire and sleep diary will begin to illuminate the sleep issues. Actigraphy, a non-invasive and objective measure of sleep, in conjunction with a sleep diary can provide a relatively simple objective measure of sleep [77] but may not be tolerated by some individuals [78••].

\section{Sleep Measurement}

Much research on sleep in individuals with a DD is based on caregiver-report or parent-report. A recent review [79••] on sleep assessment tools in children with ASD urges caution when interpreting results, which have been obtained solely by parent report, as parents are likely to focus on what they believe to be most problematic. While parents have been reported as being able to accurately report sleep latency, measures of TST, WASO, and sleep efficiency are often not as reliable [79••]. The use of multiple assessment tools including objective sleep measures such as actigraphy when investigating sleep in ASD has been emphasised [79••], and this is valid for other DDs. Videosomnography is also recommended as it is a relatively non-invasive objective measure of sleep with good reliability at detecting important sleep parameters (WASO, latency, sleep efficiency) [79••]. Where possible, self-reports of sleep are also important. Richdale and Baglin [57•] found better child-parent agreement on sleep disturbance for ASD children (70.6\%) than TD children and parents (60\%). Further, older children, adolescents and adults are less likely to disturb their care-givers' sleep, consequently, caregivers may not be aware of lengthy sleep onset delays or WASO episodes [80].

Nevertheless, parent-questionnaires or caregiverquestionnaires are important in sleep research as they can provide important information regarding behaviours surrounding sleep difficulties such as bedtime resistance and can be a valid screening tool for further assessment. However, many sleep questionnaires do not meet all 11 psychometric criteria for sound instruments, including the CSHQ and the original Simonds and Parraga questionnaire [8], and may not have been standardised for DD populations [19••]. Nevertheless, a recent comparison of the CSHQ and the MSPSQ for parent-reported sleep problems in ASD found that the MSPSQ had good internal consistency and high sensitivity and specificity, which was equal to the CSHQ [81].

Two new papers examined the use of actigraphy [78 • , 82]. Of an initial 563 older adults with ID, 179 would not wear an Actiwatch due to caregiver fears of loss or damage, or resistance or refusal by the individual with ID [78•0]. The device was successfully worn by $92.3 \%(n=382)$ of adults, but only 200 of these participants had a successful sleep measurement, mostly due to failure to use the event marker button. Depending on the sensitivity setting used for analysis, there was an hour's difference in TST and WASO, indicating that attention to sensitivity settings is needed. In order to deal with issues of compliance, the second paper [82] examined the placement of Actiwatches in custom-made shirt shoulder pockets, as compared with typical wrist placement in eight 
boys with ASD (6-10 years). Sleep latency showed best agreement for the two placements, with no systematic bias towards one placement for either latency or TST. The authors concluded that shoulder-pocket placement may be a useful alternative to obtain actigraphy measurements in children if compliance to wrist measurement is poor.

As well as sleep measurement, appropriate assessment of the sleep problem and clear practice pathways for health professionals is needed to assist the practitioner in identifying sleep issues and formulating an appropriate treatment approach. Malow and colleagues [83••] published a practice pathway for the assessment and treatment of insomnia in ASD including a free Sleep Toolkit for parents (http://www. autismspeaks.org/). Recommendations are that all children with ASD be screened for insomnia; potential medical contributions to insomnia, with treatment if they are identified; and the willingness of families to use a sleep education program teaching about sleep and behavioural approaches to intervention, followed by its implementation. For children who do not respond to this approach either medication or referral to a sleep specialist is recommended. One-year follow-up is also recommended for all children initially screened $[83 \bullet \bullet]$. This structured approach to evaluating and treating problematic sleep has much to recommend to practitioners working with individuals with DDs who present with insomnia or other symptoms of disordered sleep.

\section{Behavioural Intervention}

Common settling and night waking issues in individuals with a DD are likely to be behaviourally based $[5 \bullet \bullet, 16 \bullet \cdot$. Behavioural interventions have some empirical support [5••, $84]$ and should be based on a thorough functional assessment [85]. Several recent papers evaluated the efficacy of behavioural interventions for sleep problems in children with a DD. In the largest study [86॰], families of 80 children (2-10 years) with ASD and sleep onset insomnia participated in a multi-site sleep education program based on Reed et al. [87]. Families were randomly allocated to an individual $(1 \times 1$-hour session, two follow-up phone calls) or a group of two to four parents ( $2 \times 2$-hour sessions, two follow-up phone calls) for program delivery. The program covered sleep education and behavioural approaches to treatment, and within group sessions, individualised time for family needs. Mode of delivery did not affect outcomes. At 4-weeks post-intervention actigraphy showed significantly reduced sleep latency (18.6 minutes) across the combined delivery groups, and a significant but small improvement in sleep efficiency. All CSHQ insomniarelated subscales improved as well as improvements in child behaviour and sense of parenting competence, and postprogram evaluation showed high parent satisfaction.

A second paper reported a RCT using a 5-session, manualised behavioural parent training (BPT) program, as opposed to a parent education program about ASD, with 40 children (2-6 years) with ASD and a range of insomnia symptoms [88]. There were significant reductions by parent report on the Simonds and Parraga Composite Sleep Index scores for 15 children completing the intervention, compared with 18 children randomised to the parent education condition; however, no significant changes in actigraphy parameters occurred in either group. Parent satisfaction with both programs was high.

Three papers provided evidence of the effectiveness of individually tailored behavioural interventions to address often long-standing sleep problems in children with a DD. The effectiveness of the Sleepwise program [89], a manualised BPT program, for seven children (2-6 years) with ASD or ASD characteristics was evaluated [90]. This program consists of a pre-treatment consultation and measures, including a sleep diary; three, 2-hour parent workshops, ongoing support while treatment is implemented, and follow-up. Parents are educated about sleep, child-behaviour management, and behavioural approaches to modifying children's sleep. A sleep plan is developed and implemented for each child. Posttreatment, the CSHQ total score and night waking (sleep diary) reduced significantly, with a non-significant reduction in sleep latency. Additionally, there was a reduction in daytime behaviour problems as measured by the Developmental Behaviour Checklist (DBC) total score. Only one child did not maintain their sleep improvements at a 1-month followup: Parents approved of the program.

Five children with AS (2-11 years) received a novel behavioural treatment for persistent insomnia delivered to parents via video telehealth or phone [91•]. Following baseline sleep data collection, a 3-component intervention tailored to each child was implemented, with 1-month and 3-month follow-up. The child's sleep environment and sleep schedule, and parent-child interactions were addressed. Sleep was assessed using the abbreviated-CSHQ, a sleep diary and actigraphy with visual and statistical analysis of primary sleep and behaviour (DBC) variables. Children learned to sleep independently and behaviour (DBC) improved with these changes maintained at follow-ups. Actigraphy showed an average 30-minute increase in TST, and a 5-minute reduction in sleep latency. The CSHQ total score also reduced significantly and parents approved of the intervention.

Similarly, using a non-concurrent multiple baseline design, with functional assessment of the children's sleep problems following baseline, two children with ASD (aged 9 years) and one TD boy (aged 7 years) received individual behavioural interventions addressing their specific sleep-related behaviours [92•]. Families were provided with an individual 2hour training session to review the treatment strategy developed and regular feedback. Sleep was monitored using sleep diaries and infrared video. Sleep latency and night waking improved for all children, as did behaviours that were 
incompatible with sleep. Children's sleep goals were generally met and parents approved of the program. Additionally, the two children with ASD ceased taking sleep medication.

\section{Melatonin}

Dysregulation of the melatonin rhythm has been shown in ASD populations [93, 94], consequently, studies investigating the efficacy of melatonin in treating sleep disturbance in ASD are beginning to emerge. Meltonin has also been used to treat sleep problems in children with DDs [5••]. A recent double blind randomised controlled trial (RCT) [95 • ] of immediaterelease melatonin, beginning at $0.5 \mathrm{mg}$, titrated if necessary (maximum dose $12 \mathrm{mg}$ ), in children with DDs, including ASD, found that melatonin significantly reduced sleep latency, but not TST, WASO or sleep efficiency. Further, at the end of the 12-week trial children taking melatonin woke significantly earlier than the placebo group suggesting that melatonin may have phase advanced the sleep/wake rhythm.

A second double blind RCT [96••] using a fixed dose of $3 \mathrm{mg}$ of controlled-release melatonin randomly assigned children with ASD (4-10 years) to one of four groups (melatonin + CBT [cognitive-behavioural therapy], melatonin alone, $\mathrm{CBT}$ alone, or placebo) found a trend for the combined treatment group to experience the greatest improvement in sleep parameters. In comparing melatonin alone and CBT alone, melatonin was more effective in improving bedtime resistance, sleep onset delay, and night wakings, while CBT alone was slightly more effective in reducing sleep anxiety.

\section{Conclusions}

These new studies support previous research that both behavioural interventions and melatonin can be useful treatments for insomnia in DDs [5••]. It is not clear that an adequate functional assessment was conducted in all the behavioural intervention studies, and there was no long-term follow-up. However, these studies indicate that individualised behavioural approaches, manualised BPT programs, and phone and telehealth delivery can improve or resolve common sleep complaints associated with insomnia. The impact of treatment on daytime behaviour is less clear as the DBC (used to measure behaviour) contains questions about sleep and it is not clear that these were removed from analyses.

The melatonin studies suggest that melatonin also needs to be tailored to specific individual needs. Controlled-release melatonin is likely to be more appropriate for those individuals who have significant issues with WASO while immediate-release may be more appropriate for those with sleep onset delay problems; however, further research is needed. Further, those individuals who are experiencing co-morbid psychopathologies should also be considered for receiving CBT or other appropriate treatment for their psychological disorder. Treating sleep disturbance or psychopathology in isolation is unlikely to alleviate either of the problems as they are generally interrelated.

\section{Conclusions}

Stores' 1992 sleep review [4] drew attention to several fundamental issues concerning sleep disturbance in DD populations. These included: (1) the need to consider the clinical and psychosocial aspects of poor sleep, not just its physiological aspects; (2) separate examination of specific, clinically defined sleep disorders; (3) comparison of the different types of sleep problems with those found in TD children, as well as an examination of specific DDs; and (4) the impact of poor sleep on daytime functioning and cognition. Ten years later, Stores [32] called for professional education and recognition and treatment of sleep disturbances in at risk children, including children with a DD. Material reviewed here suggests that his $[4,32]$ recommendations remain relevant today.

Most research on sleep in DDs assesses children and younger adolescents; there is little research assessing sleep in very young children, older adolescents, or adults. Where adolescents or adults are included, it is often within samples including younger children, thus masking the developmental aspects of sleep. For example, with the onset of puberty, marked changes in sleep patterns occur [97]. Sleep disturbance in individuals with a DD can begin at an early age $[41 \cdot \bullet]$ and continue into adolescence [43•], adulthood and old age [17•]. The incidence of psychopathology during adolescence is increased, there is a high rate of anxiety and depression in individuals with ASD, and psychopathologies and sleep disturbance are associated [56, 70, 98, 99]. Similarly, health issues frequently associated with DDs may impact on sleep. Thus, more attention should be paid to the contribution of comorbid anxiety, depression, and medical conditions to poor sleep in individuals with a DD.

Although it is now emerging, there remains little research comparing specific DDs, while available research suggests that some sleep issues are more common in some disorders than in others. Children with WS and ASD appear to have sleep onset difficulties; like children with ADHD [11], children with JS have limb movements and restless sleep; and children with DS and PWS have high risk for OSA. Whether or not the aetiology of these topographically similar complaints is the same is a question for research and has implications for prevention and treatment. At present, treatment research primarily addresses insomnia and exploration of the impact of sleep problems on families is limited. The impact of variables such as behavioural and medical issues associated with DDs, family supports, and SES on children's sleep remains largely unexplored. 
Much still needs to be done in standardising our approach to understanding the aetiology and impact of sleep problems and their evaluation and treatment in DD populations. This includes examining discrete developmental disorders rather than heterogeneous samples, and specific developmental periods rather than age ranges across developmental periods. Research examining sleep in ASD has expanded significantly, but publications include reviews or tend to reinforce previous findings rather than extending our knowledge, while for other DDs research remains disappointingly sparse.

\section{Compliance with Ethics Guidelines}

Conflict of Interest Amanda L. Richdale and Emma K. Baker declare that they have no conflict of interest.

Human and Animal Rights and Informed Consent This article does not contain any studies with animal subjects performed by any of the authors. With regard to the authors' research cited in this paper, all procedures were followed in accordance with the ethical standards of the responsible committee on human experimentation and with the Helsinki Declaration of 1975, as revised in 2000 and 2008.

\section{References}

Papers of particular interest, published recently, have been highlighted as:

- Of importance

-. Of major importance

1. Bartlett L, Rooney V, Spedding S. Nocturnal difficulties in a population of mentally handicapped children. Br J Mental Subnorm. 1985;31:54-9.

2. Quine L. Sleep problems in primary school children: comparison between mainstream and special school children. Child Care Health Behav. 2001;27:201-21.

3. Didden R, Korzillius H, van Aperlo B, et al. Sleep problems and daytime problem behaviours in children with intellectual disability. J Intellect Dev Disabil. 2002;46:537-47.

4. Stores G. Annotation: sleep studies in children with a mental handicap. J Child Psychol Psychiatry. 1992;33:1303-17.

5.• Richdale AL. Autism and other developmental disabilities. In: Wolfson AR, Montgomery-Downs H, editors. The Oxford handbook of infant, child, and adolescent sleep: development and behavior. Oxford: Oxford University Press; 2013. pp. 471-94. This chapter provides an overview of sleep difficulties and treatment in a range of DDs.

6. American Psychiatric Association. Diagnostic and statistical manual of mental disorders. 5th ed. Washington DC: Author; 2013.

7. American Academy of Sleep Medicine. International classification of sleep disorders. 2nd ed. Rochester: Author; 2005.

8. Spruyt K, Gozal D. Pediatric sleep questionnaires as diagnostic or epidemiological tools: a review of currently available instruments. Sleep Med Rev. 2011;15:19-32.

9. Fernandez F, Edgin JO. Poor sleep as a precursor to cognitive decline in Down syndrome: a hypothesis. J Alzheimers Dis Parkinsonism. 2013;3(2).

10. O'Brien LM. Neurocognitive implications. In: Wolfson AR, Montgomery-Downs H, editors. The Oxford handbook of infant, child, and adolescent sleep: development and behavior. Oxford: Oxford University Press; 2013. p. 414-28.

11. Colton HR, Altevogt VM, editors. Sleep disorders and sleep deprivation: an unmet public health problem. Institute of Medicine of the National Academies. Washington DC: National Academies Press; 2006.

12. De Leersnyder H. Inverted rhythm of melatonin secretion in SmithMagenis syndrome: from symptoms to treatment. Trends Endocrinol Metab. 2006;17:291-8.

13. Chen C-C, Spanò G, Edgin JO. The impact of sleep disruption on executive function in Down syndrome. Res Dev Disabil. 2013;34: 2033-39.

14. Stores G, Stores R. Sleep disorders and their clinical significance in children with Down syndrome. Dev Med Child Neurol. 2013;55: 126-30. This paper provides a nice overview of DS and the clinical aspects of sleep in this disorder.

15. Robinson AM, Richdale AL. Sleep problems in children with an intellectual disability: parental perceptions of sleep problems, and views of treatment effectivness. J Intellect Disabil Res. 2004;30: $139-50$.

$16 . \bullet$ van de Wouw E, Evenhuis HM, Echtld MA. Prevalence, associated factors and treatment of sleep problems in adults with intellectual disability: a systematic review. Res Dev Disabil. 2012;33:1310-32. This is a comprehensive review of sleep in older adults with ID and a range of DDs, illustrating the need for work in this area.

17. van de Wouw E, Evenhuis HM, Echteld MA. Objective assessment of sleep and sleep problems in older adults with intellectual disabilities. Res Dev Disabil. 2013;34:2291-303. This provides an objective view of sleep problems and their correlates in older adults with ID.

18. Maaskant M, van de Wouw E, van Wijck R, et al. Circadian sleepwake rhythm of older adults with intellectual disabilities. Res Dev Disabil. 2013;34:1144-51.

19.•• Tietze A-L, Blankenburg M, Hechler T, et al. Sleep disturbances in children with multiple disabilities. Sleep Med Rev. 2012;16:11727. This gives an excellent overview of sleep problems and treatment in children with a $D D$, excluding ASD.

$20 . \bullet$ Ashworth A, Hill CM, Karmiloff-Smith A, Dimitriou D. Cross syndrome comparison of sleep problems in children with Down syndrome and Williams syndrome. Res Dev Disabil. 2013;34: 1572-80. This is an important paper as it is one of the few that compares sleep in two DDs as compared with a control group, using both objective and subjective measures of sleep.

21. Maas PHM, Didden R, Korzilius H, Curfs LMG. Exploration of differences in types of sleep disturbance and severity of sleep problems between individuals with $\mathrm{Cri}$ du Chat syndrome, Down's syndrome, and Jacobsen syndrome: a case control study. Res Dev Disabil. 2012;33:1773-79. This paper is interesting because of the comparison of sleep across multiple disability groups.

22. Gibbs S, Wiltshire E, Elder D. Nocturnal sleep measured by actigraphy in children with Prader-Willi syndrome. J Pediatr. 2013;162:765-9.

23. Goldman SE, Bichell TJ, Surdyka K, Malow BA. Sleep in children and adolescents with Angelman syndrome: association with parent sleep and stress. J Intellect Disabil Res. 2012, 56:600-8. This paper uses PSG, actigraphy and a sleep questionnaire to examine sleep in children with $A S$ and their parents.

24. Buckhalt JA. The role of schools in identification, treatment, and prevention of children's sleep problems. In: The Oxford handbook of infant, child, and adolescent sleep: development and behavior. Oxford: Oxford University Press; 2013. p. 292-301.

25. Hansen BH, Skirbekk B, Oerbeck B, et al. Associations between sleep problems and attentional and behavioral functioning in children with anxiety disorders and ADHD. Behav Sleep Med. 2014; 12:53-68.

26. Patil SP, Schneider H, Schwartz AR, Smith PL. Adult obstructive sleep apnea. Chest. 2007;132:325-37. 
27. Axelsson EL, Hill CM, Sadeh A, Dimitriou D. Sleep problems and language development in toddlers with Williams syndrome. Res Dev Disabil. 2013;34:3988-96.

28. Dimitriou D, Karmiloff-Smith A, Ashworth A, Hill CM. Impaired sleep-related learning in children with Williams syndrome. Pediatr Res Int J. 2013;2013. doi:10.5171/2013.662275.

29. Bonuck K, Grant R. Sleep problems and early developmental delay: implications for early intervention programs. Intellect Dev Disabil. 2012;50:41-52.

30. Buckhalt JA. Sleep and cognitive function in children with disabilities. Except Child. 2013;79:391-405.

31. Goodlin-Jones BL, Tang K, Liu J, Anders TF. Sleep patterns in pre-school age children with autism, developmental delay and typical development. J Am Acad Child Adolesc Psychiatr. 2008;47:930-8.

32. Stores G. Annotation: children's sleep disorders: modern approaches, developmental effects, and children at special risk. Dev Med Child Neurol. 2002;41:568-73.

33. Doran SM, Harvey MT, Horner RH. Sleep and developmental disabilities: assessment, treatment and outcome measures. Ment Retard. 2006;44:13-27.

34.• Vanhelst, J, Bui-Xuan G, Fardy PS, Mikulovic J. Relationship between sleep habits, anthropometric characteristics and lifestyle habits in adolescents with intellectual disabilities. Res Dev Disabil. 2013;34:2614-20. This paper is important because it examines sleep and sleep timing and relationships with weight and lifestyle, showing that this is an important consideration.

35. Fleig D, Randler C. Association between chronotype and diet in adolescents based on food logs. Eat Behav. 2009;10:115-8.

36. Hart CN, Hawley N, Kuhl E, Jelalian E. Weight control and obesity. In: Wolfson AR, Montgomery-Downs H, editors. The Oxford handbook of infant, child, and adolescent sleep: development and behavior. Oxford: Oxford University Press; 2013. p. 429-40.

37. Meltzer LJ, Mindell JA. Relationship between child sleep disturbances and maternal sleep, mood, and parenting stress: a pilot study. J Fam Psychol. 2007;21:67-73.

38. Chu J, Richdale AL. Sleep quality and psychological wellbeing in mothers of children with developmental disabilities. Res Dev Disabil. 2009;30:1512-22.

39. Gallagher S, Phillips AC, Carroll D. Parental stress is associated with poor sleep quality in parents caring for children with developmental disabilities. J Pediatr Psychol. 2010;35: 728-37.

40. Cotton S, Richdale A. Brief report: parental descriptions of sleep problems in children with autism, Down syndrome, and PraderWilli syndrome. Res Dev Disabil. 2006;27:151-61.

41.• Humphreys JS, Gringras P, Blair PS, et al. Sleep patterns in children with autistic spectrum disorders: a prospective cohort study. Arch Dis Child. 2014;99:114-18. This paper is one of the few longitudinal studies assessing sleep in children with ASD.

42. Olsson MB, Carlsson LH, Westerlund J, et al. Autism before diagnosis: crying, feeding, and sleeping problems in the first two years of life. Acta Paediatr. 2013;102:635-9.

43. Baker E, Richdale AL, Short M, Gradisar M. An investigation of sleep patterns in adolescents with high-functioning autism spectrum disorder compared with typically developing adolescents. Dev Neurorehabil. 2013;16:155-65. This paper obtains self-report measures of sleep in addition to actigraphy in a pure sample of adolescents with HFASD.

44. Goldman SE, Richdale AL, Clemons T, Malow BA. Parental sleep concerns in autism spectrum disorders: variations from childhood to adolescence. J Autism Dev Disord. 2012;42:531-8.

45. Anders T, Iosif A, Schwichtenberg AJ, et al. Sleep and daytime functioning: a short-term longitudinal study of three preschool-age comparison groups. AAIDD. 2012;117:275-90.
46. Tsai F, Chiang H, Lee C, et al. Sleep problems in children with autism, attention-deficit hyperactivity disorder, and epilepsy. Res Autism Spectr Disord. 2012;6:413-21.

47. Hiscock H, Canterford L, Ukoumunne OC, Wake M. Adverse associations of sleep problems in Australian preschoolers: national population study. Pediatrics. 2005;119:86-93.

48. Antshel KM, Hier BO. Attention deficit hyperactivity disorder (ADHD) in children with autism spectrum disorder. In: Comprehensive guide to autism. New York: Springer; 2014. p. 1013-29.

49. Taylor MA, Schreck KA, Mulick JA. Sleep disruption as a correlate to cognitive and adaptive behavior problems in autism spectrum disorders. Res Dev Disabil. 2012;33:1408-17.

50. Sikora DM, Johnson K, Clemons T, Katz T. The relationship between sleep problems and daytime behavior in children of different ages with autism spectrum disorders. Pediatrics. 2012;130:S83.

51. Tudor ME, Hoffman CD, Sweeney DP. Children with autism: sleep problems and symptom severity. Focus Autism Other Dev Disabil. 2012;27:254-62.

52. Park S, Cho S, Cho IH, et al. Sleep problems and their correlates and comborbid psychopathology of children with autism spectrum disorders. Res Autism Spect Dis. 2012;6:1068-72.

53. Meltzer LJ. Brief report: sleep in parents of children with autism spectrum disorders. J Pediatr Psychol. 2008;33:380-6.

54. Hodge D, Hoffman CD, Sweeney DP, Riggs ML. Relationship between children's sleep and mental health in mothers of children with and without autism. J Autism Dev Disord. 2013;43:956-63.

55. Tehee E, Honan R, Hevey D. Factors contributing to stress in parents of individuals with autistic spectrum disorders. J Appl Res Intellect Disabil. 2009;22:34-42.

56. Kim JA, Szatmari P, Bryson SE, et al. The prevalence of anxiety and mood problems among children with autism and Asperger syndrome. Autism. 2000;4:117-32.

57. Richdale AL, Baglin C. Self-report and caregiver-report of sleep and psychopathology in children with high-functioning autism spectrum disorder: a pilot study. Dev Neurorehabil. 2013. doi:10. 3109/17518423.2013.829534. This paper is one of the few papers to obtain self-reports of sleep and psychopathology in children with an ASD.

58. Hollway JA, Aman MG, Butter E. Correlates and risk markers for sleep disturbance in participants of the autism treatment network. J Autism Dev Disord. 2013;43:2830-43. This paper assessed the relationship between several risk factors for sleep problems and sleep in a large sample of children with ASD.

59.• Limoges E, Bolduc C, Berthiaume C, et al. Relationship between poor sleep and daytime cognitive performance in young adults with autism. Res Dev Disabil. 2013;34:1322-35. This paper is one of the few studies to assess the impact of poor sleep on daytime cognitive performance in adults with ASD.

60. Jan JE, Owens JA, Weiss MD, et al. Sleep hygiene for children with neurodevelopmental disabilities. Pediatrics. 2008;122:143-1350.

61. Englehardt CR, Mazurek MO, Sohl K. Media use and sleep among boys with ASD, ADHD or TD. Pediatrics. 2013;132:1081-89. This paper is important as it assesses the impact of in-room media systems in boys with ASD, ADHD and TD. It emphasises the need for good sleep hygiene in boys with ASD.

62. Gradisar M, Short MA. Sleep hygiene and environment: role of technology. In: Wolfson AR, Montgomery-Downs H, editors. The Oxford handbook of infant, child, and adolescent sleep: development and behavior. Oxford: Oxford University Press; 2013. p. $113-26$.

63. Youssef J, Singh K, Huntington N, et al. Relationship of serum ferritin levels to sleep fragmentation and periodic limb movements of sleep on polysomnography in autism spectrum disorders. Pediatr Neurol. 2013;49:274-8. 
64. Dosman CF, Brian JA, Drmic IE, et al. Children with autism: effect of iron supplementation on sleep and ferritin. Pediatr Neurol. 2007;36:152-8.

65. Cortese S, Konofal E, Bernardina BD, et al. Sleep disturbances and serum ferritin levels in children with attention-deficit/hyperactivity disorder. Eur Child Adolesc Psychiatry. 2009;18:393-9.

66. Chaidez V, Hansen RL, Herz-Picciotto I. Gastrointestinal problems in children with autism, developmental delays or typical development. J Autism Dev Disord. 2013. doi:10.1007/s10803-013-1973-x.

67. Mannion A, Leader G, Healy O. An investigation of comorbid psychological disorders, sleep problems, gastrointestinal symptoms and epilepsy in children and adolescents with Autism Spectrum Disorder. Res Autism Spect Disord. 2013;7:35-42.

68. Corbett BA, Mendoza S, Wegelin JA, et al. Variable cortisol circadian rhythms in children with autism and anticipatory stress. J Psychiatr Neurosci. 2008;33:227-34.

69. Richdale AL, Prior MR. Urinary cortisol circadian rhythm in a group of high-functioning children with autism. J Autism Dev Disord. 1992;22:433-47.

70. Gregory AM, Sadeh A. Sleep, emotional and behavioral difficulties in children and adolescents. Sleep Med Rev. 2012;16:129-36.

71. Reynolds S, Lane SJ, Thacker L. Sensory processing, physiological stress, and sleep behaviours in children with and without autism spectrum disorders. OTJR Occup Particip Health. 2012;32:246-57. This paper is important as it examines the association between physiologic responses to sensation and sleep in children with ASD.

72. Grønborg TK, Schendel DE, Parner ET. Recurrence of autism spectrum disorders in full- and half-siblings and trends over time. A population-based cohort study. JAMA Pediatr. 2013;167:947-53

73. Schwitenberg AJ, Young GS, Hutman T, et al. Behavior and sleep problems in children with a family history of autism. Autism Res. 2013;6:169-76. This paper is important as it details sleep and behaviour problems experienced by high and low risk siblings for ASD.

74. Chou M, Chou W, Chiang H, et al. Sleep problems among Taiwanese children with autism, their siblings and typically developing children. Res Autism Spectr Dis. 2012;6:665-72.

75. Kotagal S, Broomall E. Sleep in children with autism spectrum disorders. Pediatr Neurol. 2012;47:242-51.

76. Richdale AL, Schreck KA. Sleep problems in autism spectrum disorders: prevalence, nature possible biopsychosocial aetiologies. Sleep Med Rev. 2009;13:403-11.

77. Horne RSC, Biggs SN. Actigraphy and sleep/wake diaries. In: Wolfson AR, Montgomery-Downs H, editors. The Oxford handbook of infant, child, and adolescent sleep: development and behavior. Oxford: Oxford University Press; 2013. p. 189-203.

78.• Van Dijk E, Hilgenkamp TIM, Evenhuis HM, Echteld MA. Exploring the use of actigraphy to investigate sleep problems in older people with intellectual disability. J Intellect Disabil Res. 2012;56:204-11. This paper has some important practice points for the use of actigraphy in older adults with ID.

79.• Hodge D, Parnell AMN, Hoffman CD, Sweeney DP. Methods for assessing sleep in children with autism spectrum disorders: a review. Res Autism Spectr Disord. 2012;6:1337-44. This paper provides a good overview of the methods used for assessing sleep in ASD populations.

80. Sadeh A. Commentary: comparing actigraphy and parental report as measures of children's sleep. J Pediatr Psychol. 2008;33:406-7.

81. Johnson CR, Turner KS, Foldes E, et al. Comparison of sleep questionnaires in the assessment of sleep disturbances in children with autism spectrum disorders. Sleep Med. 2012;13:795-801.

82. Adkins KW, Goldman SE, Fawkes D, et al. A pilot study of shoulder placement for actigraphy in children. Behav Sleep Med. 2012;10:138-47.

83.• Malow BA, Byars, K, Johnson K, et al. A practice pathway for the identification, evaluation and management of insomnia in children and adolescents with autism spectrum disorders. Pediatrics. 2012;130:S106-24. A useful practice model for clinicians working with children with sleep problems.

84. Turner KS, Johnson CR. Behavioral interventions to address sleep disturbances in children with autism spectrum disorder: a review. Top Early Child Spec Educ. 2013;33:144-52.

85. Blampied NM. Functional behavioural analysis of sleep in infants and children. In: The Oxford handbook of infant, child, and adolescent sleep: development and behavior. Oxford: Oxford University Press; 2013. p. 169-88.

86. Malow BA, Adkins KW, Reynolds A, et al. Parent-based sleep education for children with autism spectrum disorders. J Autism Dev Disord. 2014;44:216-228. Indicates that small group parent sleep education programs can address sleep onset delay.

87. Reed HE, McGrew SG, Artibee K, et al. Parent-based sleep education workshops in autism. J Child Neurol. 2009;24:93645 .

88. Johnson CR, Turner KS, Foldes E, et al. Behavioral parent training to address sleep disturbances in young children with autism spectrum disorder: a pilot trial. Sleep Med. 2013;14:995-1004.

89. Intellectual Disability Services Council. Sleepwise: a resource manual. Positive sleep practices for young children with developmental delay. Adelaide: Department for Families and Communities; 2005.

90. Austin KL, Gordon JE, O'Connell A. Preliminary evaluation of Sleepwise program for children with sleep disturbance and developmental delay. Child Fam Behav Ther. 2013;35:195-211.

91. Allen KD, Kuhn BR, DeHaai KA, Wallace DP. Evalution of a behavioral treatment package to reduce sleep problems in children with Angelman syndrome. Res Dev Disabil. 2013;34:676-86. Shows that the careful application of tailored behaviour interventions can be delivered via telehealth or phone.

92. Jin CS, Hanley GP, Beaulieu L. An individualised and comprehensive approach to treating sleep problems in young children. JABA. 2013;46:161-80. Illustrates the effectiveness of a careful functional assessment and application of an individualised behavioural sleep treatment.

93. Melke J, Goubran Botros H, Chaste P, et al. Abnormal melatonin synthesis in autism spectrum disorders. Mol Psychiatr. 2008;13:908 .

94. Tordjman S, Anderson GM, Bellissant E, et al. Day and nighttime excretion of 6-sulphatoxymelatonin in adolescents and young adults with autistic disorder. Psychoneuroendocrinology. 2012;37: 1990-97.

95.• Gringras P, Gamble C, Wiggs L, et al. Melatonin for sleep problems in children with neurodevelopmental disorders: randomised double masked placebo controlled trial. BMJ. 2012;345:e6664. This RCT is important as it assesses the use of immediate-release melatonin to treat sleep problems in children with various neurodevelopmental disorders.

96.• Cortesi F, Giannotti F, Sebastiani T, et al. Controlled-release melatonin, singly and combined with cognitive behavioural therapy, for persistent insomnia in children with autism spectrum disorders: a randomized placebo-controlled trial. J Sleep Res. 2012;21:700-9. This paper shows that controlled-release melatonin in conjunction with CBT may be beneficial in treating persistent insomnia in children with ASD.

97. Gradisar M, Gardner G, Dohnt H. Recent worldwide sleep patterns and problems during adolescence: a review and meta-analysis of age, region, and sleep. Sleep Med. 2011;12:110-8.

98. Gregory AM, Buysse DJ, Willis TA, et al. Associations between sleep quality and anxiety and depression symptoms in a sample of young adult twins and siblings. J Psychosom Res. 2011;71:250-5.

99. Ivanenko A, Johnson K. Sleep disturbance in children with psychiatric disorders. Seminars Pediatr Neurol. 2008;15:70-8. 